\title{
FANSY 1.0: a phenomenological model for simulation of coplanar particle generation in superhigh-energy hadron interactions
}

\author{
Rauf Mukhamedshin ${ }^{\mathrm{a}}$ \\ Institute for Nuclear Research, Russian Academy of Sciences, Moscow 117312, Russia
}

Received: 17 April 2008 / Revised: 21 December 2008 / Published online: 26 February 2009

(C) Springer-Verlag / Società Italiana di Fisica 2009

\begin{abstract}
Simulations show that a phenomenon of coplanarity of most energetic subcores of $\gamma$-ray-hadron families found in mountain-based and stratospheric X-ray-emulsion chamber experiments requires to introduce a coplanar particle generation with large transverse momenta in hadron interactions at superhigh energies. Some physical mechanisms are considered. A phenomenological model, which makes it possible to simulate the coplanar particle generation, is presented. Different versions of this model are considered, their features are described and compared with those of models applied by the CORSIKA package. Cosmic-ray experimental data and simulated results are compared. Conclusion on features of hadron interactions at superhigh energies and some predictions with respect to LHC experiments are made.
\end{abstract}

PACS $02.70 . \mathrm{Uu} \cdot 12.90 .+\mathrm{b} \cdot 13.85 .-\mathrm{t}$

\section{Introduction}

Experiments with extensive air showers (EAS) have formed an opinion that soft strong interactions of protons and nuclei of the primary cosmic radiation (PCR) at superhigh $\left(E_{0} \gtrsim 10^{16} \mathrm{eV}\right.$ ) energies are rather well-described with a concept presented by quark-gluon string models (QGSM). On the other hand, such a robust and transparent phenomenon is observed at these energies as a tendency for a coplanarity of most energetic cores of so called $\gamma$-ray-hadron families, i.e., groups of highest-energy $(E \gtrsim n \cdot 1 \mathrm{TeV})$ particles in EAS cores). The effect has been first found by the Pamir Collaboration in X-ray-emulsion chamber (XREC) experiments [1-5] and confirmed later in other mountain [6] and stratospheric [7-10] experiments. While assuming this observable effect to be produced by well-known elementary

a e-mail: muhamed@sci.lebedev.ru particles, it is related to hadron-nucleus interactions at superhigh energies $\left(E_{0} \gtrsim 10^{16} \mathrm{eV}\right.$ [11], i.e., $\sqrt{s} \gtrsim 4 \mathrm{TeV}$ ) and can be characterized by large average transverse momenta, for instance, up to $\sim n \times 10 \mathrm{GeV} / c$ as early as at $E_{0} \sim 10^{16} \mathrm{eV}[12,13]$.

There is a rather widespread point of view that this effect is not more than a tail in a distribution caused by fluctuations. This opinion is mainly based, on the one hand, on the fact that collider experiments do not show an evidence for exotic types of interactions. On the other hand, it is caused by desultory and fragmentary awareness based, as a rule, on separated results presented by different experimental groups with different type X-ray emulsion chambers (XREC), namely, with so called "carbon", "lead", "iron" and other ones (C-, Pb-, Fe-XREC and so on). However, to analyze the problem as a whole, it is necessary to take into account the following important points.

1. There are five independent sets of experimental data, namely, data accumulated by mountain-based Pamir's $\mathrm{C}$ - and $\mathrm{Pb}$-XRECs (including events with observed energies of electromagnetic particles, i.e., $\gamma$-rays, $e^{ \pm}$) $\left.\sum E_{\gamma} \geq 700 \mathrm{TeV}\right)$, Mt.Canbala's Fe-XRECs $\left(\sum E_{\gamma} \geq\right.$ $500 \mathrm{TeV})$ as well as two highest-energy stratospheric events (Strana and JF2af2) with $\sum E_{\gamma} \gtrsim 1400 \mathrm{TeV}$ each. All these data are related to hadron-nucleus interaction energies $E_{0} \gtrsim 10^{16} \mathrm{eV}$ unachievable for accelerators and colliders during several decades.

2. All the mountain experimental XREC data sets demonstrate an excess of the fraction of coplanar events as compared with calculated background values. Both stratospheric events being unique in energy are unique in extreme coplanarity as well.

3. To calculate statistical errors of mountain-level data on fraction of coplanar events, it is more correct to apply the binomial distribution, accounting for division of a limited-number events into two classes (in our case, aligned families and not aligned ones), and not the Pois- 
son distribution. This approach decreases the statistical errors as compared with published ones.

4. While calculating the probability that the above-mentioned sets of experimental data are caused by fluctuations, we get the following values of probability for each of the data sets, namely, for Pamir's Pb-XREC, Pamir's C-XREC, Mt.Kanbala's Fe-XREC, the Strana and JF2af2 events: $9 \times 10^{-5}, 1.5 \times 10^{-4}, 9 \times 10^{-2}$, $3 \times 10^{-3},<10^{-9}$, respectively [14]. As these data sets are statistically independent, one can qualitatively estimate the magnitude of a total probability $W_{\text {tot }}^{\text {fluct }}$ for the five above-mentioned experimental results to be produced by cascade-development fluctuations by multiplying single probability values. If so, $W_{\text {tot }}^{\text {fluct }} \lesssim 10^{-20}$ [14]. Thus, the explanation of the alignment phenomenon with trivial fluctuations seems to be actually improbable.

The phenomenon under consideration is related to energies which are unachievable yet with the use of accelerators. Only the LHC will provide energies required to test this phenomenon and, may be, give an information to make this problem more clear (see Sect. 4.4). As regards EAS experiments, this phenomenon cannot be studied due to a rather poor coordinate and energy resolution of detectors aimed at measurements of the total charged-particle number rather than to study the fine high-energy structure of the EAS' central core (see Sect. 4.3).

This effect is to be taken into account both in LHC experiments (as a high- $p_{t}$ background, at least) and especially in ground-based superhigh-energy cosmic-ray and astrophysical experiments. In the latter case just the lateral features of air cascades being strongly influenced by transverse momentum are used to obtain information on the PCR's nuclear composition at superhigh energies.

As is shown in [14], the coplanarity phenomenon is

- not described by QGSMs;

- not explained in the QCD framework;

- characterized by a cross section being comparable with the proton's inelastic one;

- related to most energetic secondary particles generated in hadron interactions;

- connected with a specific correlation between longitudinal $\left(p_{L}\right)$ and transverse $\left(p_{t}^{\mathrm{copl}}\right)$ momenta of particles in the coplanarity plane: the lower is $p_{L}$, the higher is $p_{t}^{\text {copl }}$.

There are a few theoretical ideas related to this phenomenon, namely, the conservation of the angular momentum of a relativistic fast-rotating quark-gluon string (QGS) stretched between colliding hadrons (Fig. 4d in [14]) [15], semihard double diffraction dissociation, which assumes the coplanarity to be a result of a QGS tension in the diffraction cluster between a semihardly scattered constituent quark and other spectator quarks of the projectile hadron and its following rupture (Fig. $4 \mathrm{~b}$ in [14]) [16]; projectile's diquark breaking [17]; appearance of very-high-spin leading systems $[18,19]$. This problem seems to be likely resolved by using the first two approaches.

This work is inspired by the concept [15]. In this case the coplanarity of secondaries is not exotic one but a rather natural consequence of the angular-momentum conservation law and appears as a simple kinematic effect with a cross section being comparable with the usual inelastic one. As this concept is proposed in general terms only, we can solely apply a phenomenological approach to design a model to analyze experimental data.

As the detailed mechanism of transformation of the QGS angular momentum into transverse momenta of particles is unknown, we consider below four versions of $p p$ and $p N$ interactions of the model named FANSY (FAN-like Secondary particle Yield), namely, a traditional $Q G S J$ version, which does not include unusual processes, as well as very weak (feeble), moderate (weak), and extremist (strong) versions of coplanar particle generation (CPG).

General features of all the versions (multiplicity, cross section and so on) are the same, as well as all the longitudinal characteristics being identical in terms of longitudinal momentum, $p_{Z}$, as well as in relative units, $x_{\mathrm{F}} \simeq x_{\mathrm{Lab}}=$ $p_{Z} / p_{0}$, both in center-of-mass system (c.m.s.) and Lab reference frame (excluding a very small difference caused by energy and momentum conservation laws).

The versions differ in terms of transverse momentum and pseudorapidity only, for instance, in energy dependence of average and maximum values of the coplanar transverse momentum, $\left\langle p_{t}^{\mathrm{copl}}\left(E_{0}\right)\right\rangle$ and $\left\langle p_{t \text { max }}^{\mathrm{copl}}\left(E_{0}\right)\right\rangle$. The belowconsidered FANSY 1.0 is assumed to be improved in future in accordance with new experimental and theoretic achievements.

All below-given data related to CORSIKA package's models [20] are taken from talks presented at VIHKOS CORSIKA School 2005 [21].

The paper is organized as follows. Sect. 2 presents main features of the traditional $Q G S J$ version. Specific features of different versions related to simulation of coplanar particle generation are presented in Sect. 3. Comparison with experimental data is discussed in Sect. 4. In doing so, air shower development model testing is made in Sect. 4.1, comparison with XREC data is given in Sect. 4.2, EAS-related problems are discussed in Sect. 4.3, and problems related to LHC experiments are considered in Sect. 4.4. Final comments are given in Conclusion.

\section{FANSY/QGSJ: a traditional version}

The basic FANSY/QGSJ version is a model improving a completely original QGSM-oriented MC0 code [22], which is the basic code of the Pamir Collaboration and describes 
well the totality of its data [23-25] at hadron interaction energies of $\sqrt{s} \lesssim 4 \mathrm{TeV}$. Both FANSY and MC0 are phenomenological models, which take into account the soft particle generation including diffraction processes and added by generation of semihard and hard jets. The majority of FANSY/QGSJ's parameters is advisedly placed between those of QGSJET II and SIBYLL 2.1 models exploited by the CORSIKA package and most often applied to compare EAS experimental simulated data.

To simulate generation of soft-interaction particles, both FANSY and MC0 use empirical probabilities for each particle kind to be generated in parallel with energy, momentum, charge, baryon number conservation laws. Single (SD) and double diffraction (DD) processes are taken into account in pp interactions additionally to non-single-diffraction (NSD) particle generation. All these processes (excluding DD) are taken into account in $p N$ interactions as well. Jet generation is possible in SD processes at sufficiently high diffractioncluster masses.

NSD and jet generation are simulated in parallel. As regards the jet simulation, hard parton cascade development and soft hadronization are taken into account. In doing so, quark statistics is used.
Relative-momentum ( $x_{\mathrm{F}}$ and $x_{\mathrm{Lab}}$ ) distributions of particles are very important model characteristics as just these ones determine the EAS development and observed features used in ground-based experiments to study astrophysical and high-energy physics problems. Unfortunately, these parameters are difficult to study at colliders. So, corresponding accelerator data are actually absent as early as at $E_{0} \gtrsim$ $10^{12} \mathrm{eV}$ in $p p$ and $p N$ interactions. Among these characteristics just $x_{\mathrm{F}}$ and $x_{\mathrm{Lab}}$ distributions of the most energetic hadron in each interaction (baryon in $p p$ and $p N$ interactions) are most important with respect to the cascade development. Figure 2.1 shows Lab-system $d N / d x_{\text {Lab }}$ distributions for the most energetic baryon (denoted m.e.b. below) generated in $p p$ interactions at $E_{0}=10^{15}$ and $10^{19} \mathrm{eV}$ in the framework of FANSY 1.0 and different models used by the CORSIKA package [20]. Corresponding m.e.b. $d N / d x_{\text {Lab }}$ distributions in $p N$ interactions at the same energies realized by the same models are shown in Fig. 2.2. Obviously, $p N$-interaction distributions are more steep as compared with $p p$ interactions. A wide scattering of distributions realized by models illustrates the actual state of affairs in theoretical description of soft interactions.

Fig. 2.1 M.e.b. $d N / d x_{\text {Lab }}$ distributions at

(a) $E_{0}=10^{15} \mathrm{eV}$ and

(b) $E_{0}=10^{19} \mathrm{eV}$ in $p p$ interactions simulated by CORSIKA models and FANSY 1.0
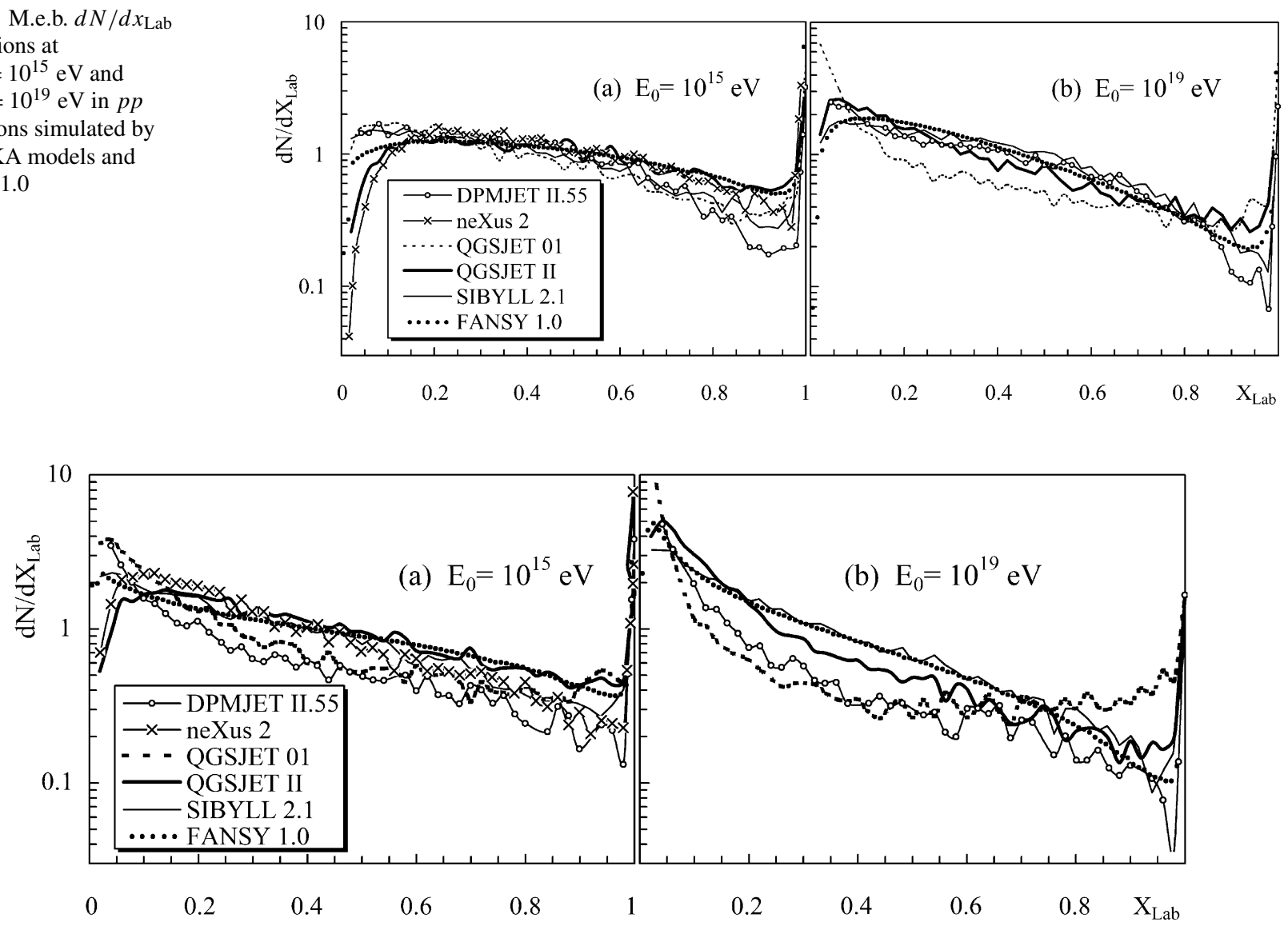

Fig. 2.2 M.e.b. $d N / d x_{\text {Lab }}$ distributions at (a) $E_{0}=10^{15} \mathrm{eV}$ and (b) $10^{19} \mathrm{eV}$ in $p N$ interactions simulated by CORSIKA models and FANSY 1.0 
Figures 2.3 and 2.4 shows m.e.b. c.m.s. $d N / d x_{\mathrm{F}}$ distributions in $p p$ interactions and Lab $d N / d x_{\mathrm{Lab}}$ distributions in $p N$ interactions, respectively, simulated by FANSY 1.0 at $E_{0}=10^{12}, 10^{15}, 10^{17}, 10^{19}$, and $10^{20} \mathrm{eV}$. One can see some steepening of distributions with increasing energy that means some decrease of average values of m.e.b. $d N / d x_{\mathrm{F}}$ and $d N / d x_{\mathrm{Lab}}$ distributions, i.e., $\left\langle x_{\mathrm{F}}\right\rangle$ and $\left\langle x_{\mathrm{Lab}}\right\rangle$, respectively. This effect is demonstrated in Fig. 2.5, where energy dependences of $\left\langle x_{\mathrm{Lab}}\right\rangle$ in $p N$ interactions simulated by different CORSIKA package's models and FANSY 1.0 are shown. An evident scattering of the curves given by models cannot be unfortunately tested with collider experiments in any case.

Pseudorapidity is a parameter being more important for accelerator experiments than for cosmic ray ones which deal with cascade development effects determined mainly by $x_{\text {Lab }}$ distributions. However, this parameter is traditionally used to compare different model and experimental accelerator data. C.m.s. charged-particle $d n_{\mathrm{ch}} / d \eta$ distributions in $p p$ interactions at $E_{0}=10^{19} \mathrm{eV}$ simulated by CORSIKA

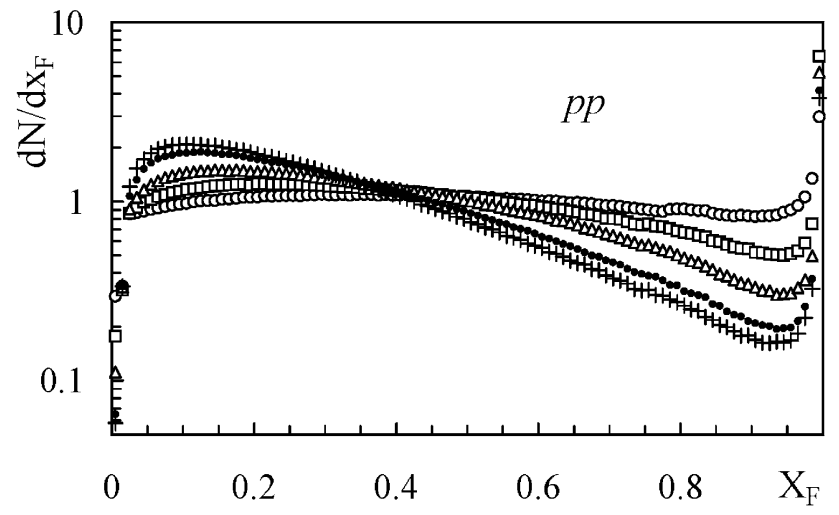

Fig. 2.3 C.m.s. m.e.b. $d N / d x_{\mathrm{F}}$ distributions in $p p$ interactions simulated by FANSY 1.0 at $E_{0}=10^{12}(O), 10^{15}(\square), 10^{17}(\triangle), 10^{19}(\bullet)$, and $10^{20}(+) \mathrm{eV}$

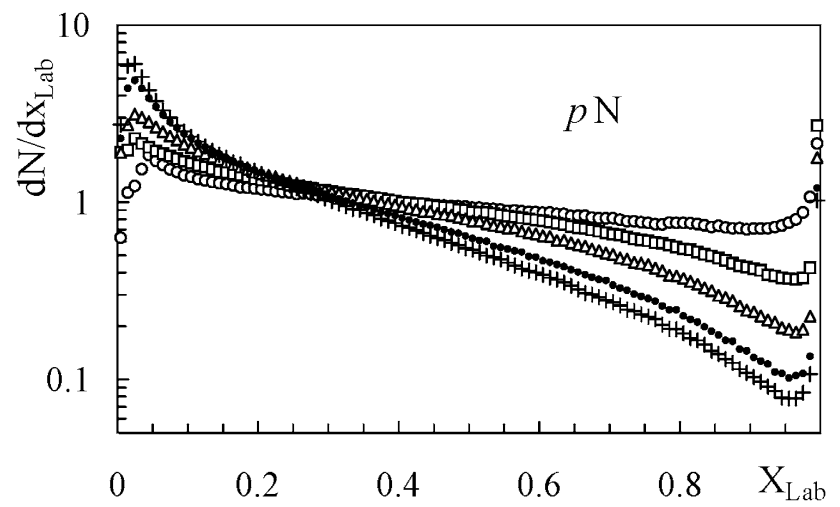

Fig. 2.4 Lab m.e.b. $d N / d x_{\text {Lab }}$ distributions in $p N$ interactions simulated by FANSY 1.0 at $E_{0}=10^{12}(O), 10^{15}(\square), 10^{17}(\triangle), 10^{19}(\bullet)$, and $10^{20}(+) \mathrm{eV}$ models and FANSY/QGSJ are shown in Fig. 2.6. Some rather strong spreading of curves is naturally related to differences in multiplicities and transverse-momentum distributions simulated by these models.

Figure 2.7 presents energy dependences of average transverse momentum of positive pions, $\left\langle p_{t}^{\pi^{+}}\left(E_{0}\right)\right\rangle$, measured in experiment and realized by different models including FANSY/QGSJ. Obviously, models demonstrate rather different energy dependences. Sometimes, their behavior even do not agree with experimental data at low energies. For instance, GEISHA 2002 shows decreasing $\left\langle p_{t}\right\rangle$, while QGSJET 01 demonstrates too strong energy dependence of $\left\langle p_{t}\right\rangle$ as compared with experimental data.

Generation of semihard and QCD jets in MCO and FANSY models is illustrated by Fig. 2.8, which presents transverse energy distributions in $p \bar{p}$ interactions at $\sqrt{s}=$ $540 \mathrm{GeV}$ and $\eta=0$ both measured by UA1 experiment and simulated data.

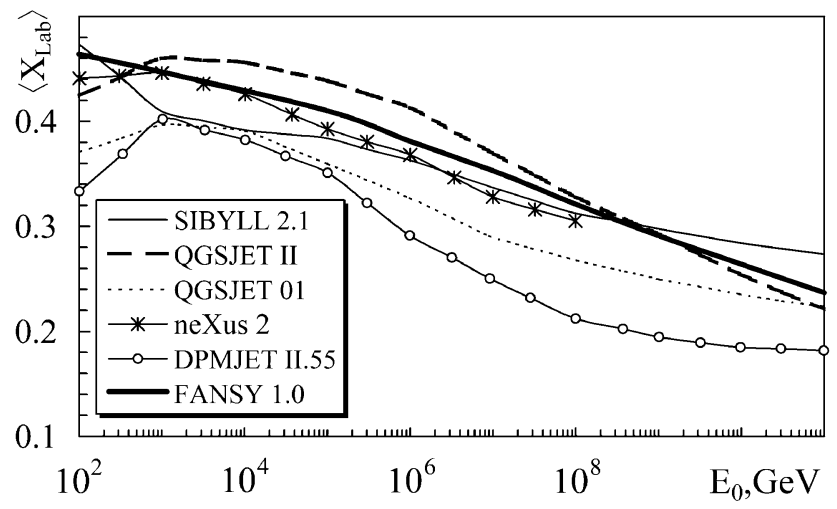

Fig. 2.5 Energy dependence of m.e.b. $\left\langle x_{\mathrm{Lab}}\right\rangle$ in $p N$ interactions simulated by CORSIKA package's models and FANSY 1.0

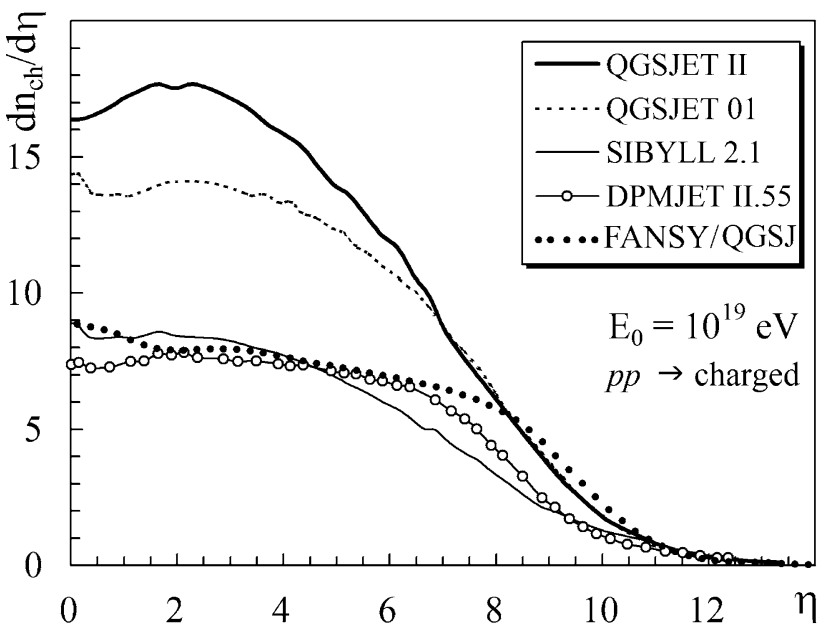

Fig. 2.6 C.m.s. charged-particle pseudorapidity distributions, $d n_{\mathrm{ch}} / d \eta$, in $p p$ interactions simulated by CORSIKA models and FANSY $/ Q G S J$ at $E_{0}=10^{19} \mathrm{eV}$ 
Average multiplicity per one interaction is a traditional parameter measured in accelerator experiments. As regards the EAS development, the influence of low-energy particles, which give the major contribution into this parameter, is not highly important. However, it is a parameter used for

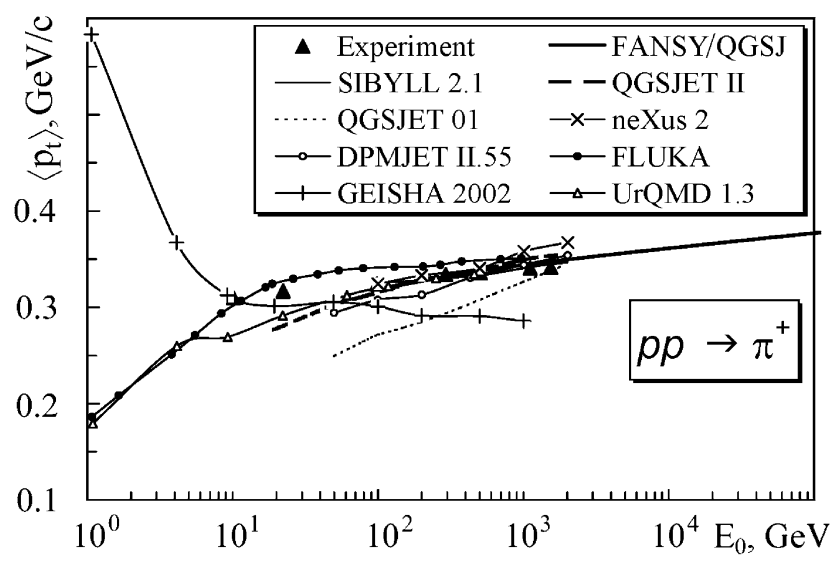

Fig. 2.7 Experimental and model energy dependences of average transverse momentum of positive pions, $\left\langle p_{t}^{\pi^{+}}\left(E_{0}\right)\right\rangle$, in $p p$ interactions

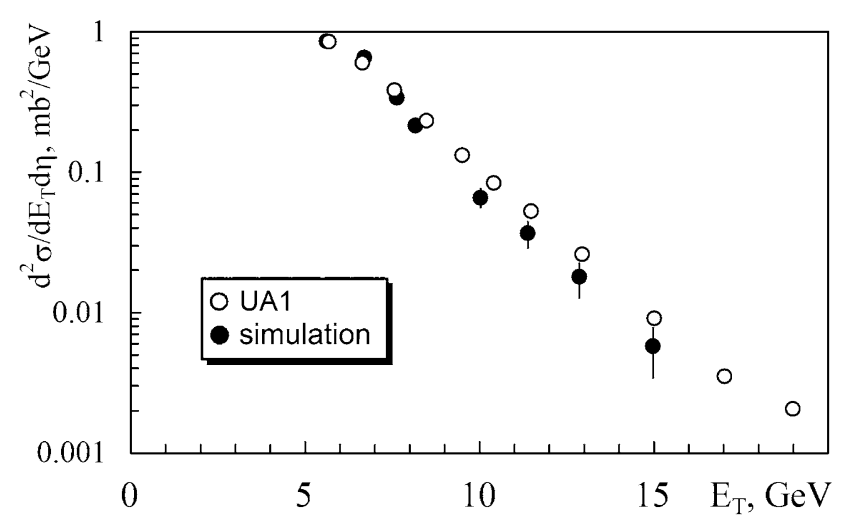

Fig. 2.8 Experimental and simulated transverse-energy distributions in $p \bar{p}$ interactions at $\sqrt{s}=540 \mathrm{GeV}$ and $\eta=0$

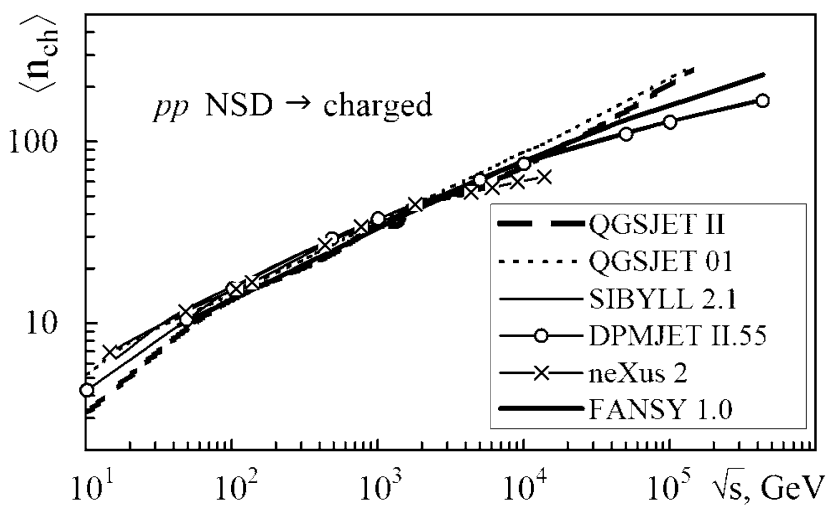

Fig. 2.9 Model energy dependences of charged-particle multiplicity, $\left\langle n_{\mathrm{ch}}(\sqrt{s})\right\rangle$, in $p p$ NSD interactions comparison of models. Energy dependence of the chargedparticle multiplicity in $p p$ NSD interactions, $\left\langle n_{\mathrm{ch}}(\sqrt{s})\right\rangle$, and $p N$ interactions, $\left\langle n_{\mathrm{ch}}\left(E_{0}\right)\right\rangle$, is presented in Figs. 2.9 and 2.10 , respectively.

FANSY 1.0 accounts for a number of types of stable and unstable particles. Multiplicities of main types of stable $\left(\tau_{0}>10^{-15} \mathrm{~s}\right)$ particles and dispersions of charged particles in $p p$ NSD interactions at $\sqrt{s}=0.0433-433 \mathrm{TeV}$ $\left(E_{0}=10^{12}-10^{20} \mathrm{eV}\right)$ are presented in Table 2.1. A part of these particles is generated through decay of meson and

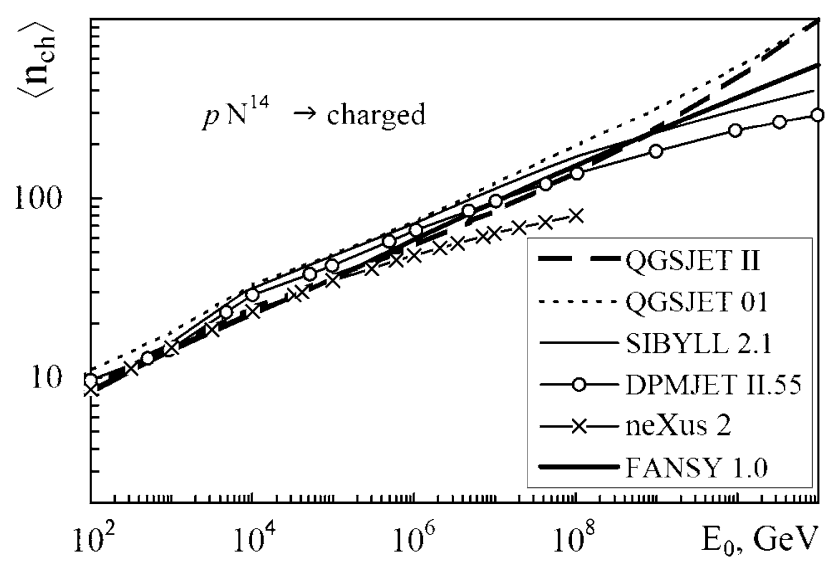

Fig. 2.10 Model energy dependence of charged-particle multiplicity, $\left\langle n_{\mathrm{ch}}\left(E_{0}\right)\right\rangle$, in $p N$ interactions

Table 2.1 Multiplicities of particles and dispersions of charged particles realized by FANSY 1.0 in $p p$ NSD interactions at $\sqrt{s}=0.0433$, $0.54,13.24$ and $433 \mathrm{TeV}$

\begin{tabular}{|c|c|c|c|c|}
\hline \multirow{2}{*}{$\begin{array}{l}\text { Type of } \\
\text { particle }\end{array}$} & \multicolumn{4}{|c|}{$\sqrt{s}, \mathrm{TeV}$} \\
\hline & 0.0433 & 0.54 & 13.24 & 433 \\
\hline$p$ & 1.411 & 1.488 & 2.62 & 5.91 \\
\hline$n$ & 0.510 & 0.587 & 1.73 & 5.02 \\
\hline $\bar{p} / \bar{n}$ & 0.006 & 0.136 & 2.36 & 8.93 \\
\hline$\Lambda^{0}$ & 0.085 & 0.130 & 0.71 & 2.28 \\
\hline $\bar{\Lambda}^{0}$ & 0.0016 & 0.039 & 0.57 & 2.02 \\
\hline$\Lambda_{c}^{ \pm}$ & 0.0061 & 0.0191 & 0.199 & 0.673 \\
\hline$\Sigma^{ \pm}$ & 0.0032 & 0.0200 & 0.239 & 0.834 \\
\hline $\bar{\Sigma}^{ \pm}$ & 0.0004 & 0.0094 & 0.146 & 0.541 \\
\hline$\pi^{ \pm}$ & 7.646 & 22.30 & 71.2 & 187.8 \\
\hline$K^{ \pm}$ & 0.541 & 2.181 & 9.45 & 27.0 \\
\hline$K_{S, L}^{0}$ & 0.424 & 1.920 & 8.70 & 25.26 \\
\hline$D^{ \pm}$ & 0.0027 & 0.0276 & 0.185 & 0.597 \\
\hline $\bar{D}^{0} / D^{0}$ & 0.0055 & 0.0342 & 0.201 & 0.668 \\
\hline$e^{ \pm}$ & 0.102 & 0.290 & 1.00 & 2.65 \\
\hline$\gamma$ & 8.504 & 24.24 & 83.7 & 221.6 \\
\hline$\left\langle n_{\mathrm{ch}}\right\rangle$ & 9.72 & 26.6 & 86.2 & 229.4 \\
\hline$D$ & 4.66 & 12.6 & 33.4 & 75.4 \\
\hline
\end{tabular}


baryon resonances $\left(\rho^{ \pm, 0}, \omega, K^{* \pm, 0}, D^{* \pm, 0}, \Delta^{++}\right.$and other particles). Fluctuations of the number of secondaries is determined by contributions of different channels (fluctuations of $K_{\text {inel }}$, numbers of semihard jets, quark-gluon strings, secondaries per QGS and so on).

Figure 2.11 displays c.m.s. $x_{\mathrm{F}} d F / d x_{\mathrm{F}}$ distributions for some kinds of particles $\left(p, n, \pi^{ \pm}, K^{ \pm}\right.$etc.) realized by FANSY 1.0 in $p p$ interactions at $E_{0}=10^{12}, 10^{17}$, and $10^{20} \mathrm{eV}$. Figure 2.12 shows corresponding Lab distributions realized in $p N$ interactions. One can see some softening of particle spectra with increasing energy. Some relative hardening of spectra of pions and kaons at $x_{\mathrm{F}} \gtrsim 0.6$ is caused by diffractive processes.
One of the most important interaction parameters of any model applied in simulation of showers in the Earth's atmosphere is the behavior of production cross section of different-type hadrons at highest energies. Figure 2.13 shows $p$-air, $\pi$-air, and $K$-air production cross sections vs. energy used by FANSY and other models. One can see a strong spreading of curves applied by different models at superhigh energies.

FANSY's cross section values shown in Fig. 2.13 include single-diffraction cross section ones. As regards $p N$ interactions, $\sigma_{\mathrm{SD}}$ increases slowly from 24 mbarn at $E_{0}=10^{11} \mathrm{eV}$ to $29.1 \mathrm{mbarn}$ at $E_{0}=10^{20} \mathrm{eV}$.

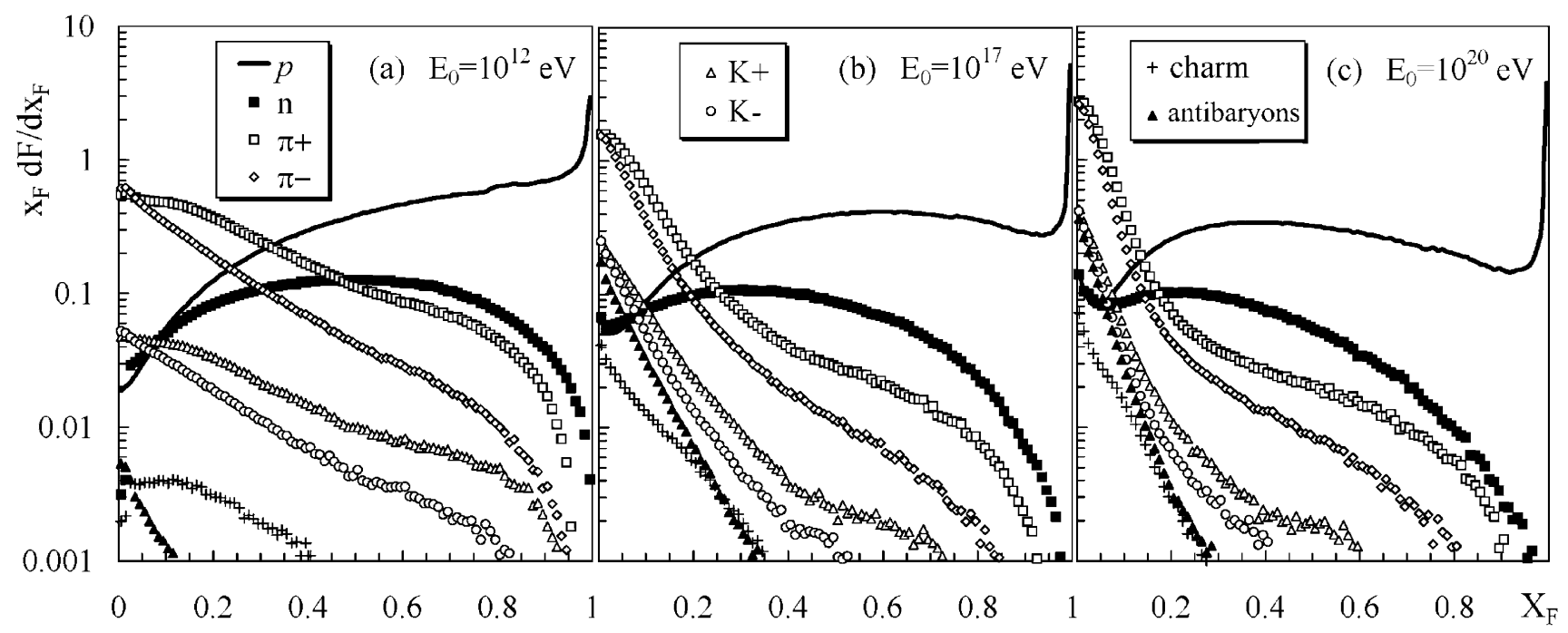

Fig. 2.11 C.m.s. $x_{\mathrm{F}} d F / d x_{\mathrm{F}}$ distributions simulated by FANSY 1.0 in $p p$ interactions at energies of (a) $10^{12}$, (b) $10^{17}$, (c) $10^{20} \mathrm{eV}$ for protons (curve), neutrons $(\mathbf{\square}), \pi^{+}(\square), \pi^{-}(\diamond), K^{+}(\triangle), K^{-}(\bigcirc)$, charmed particles (+), antibaryons $(\Delta)$

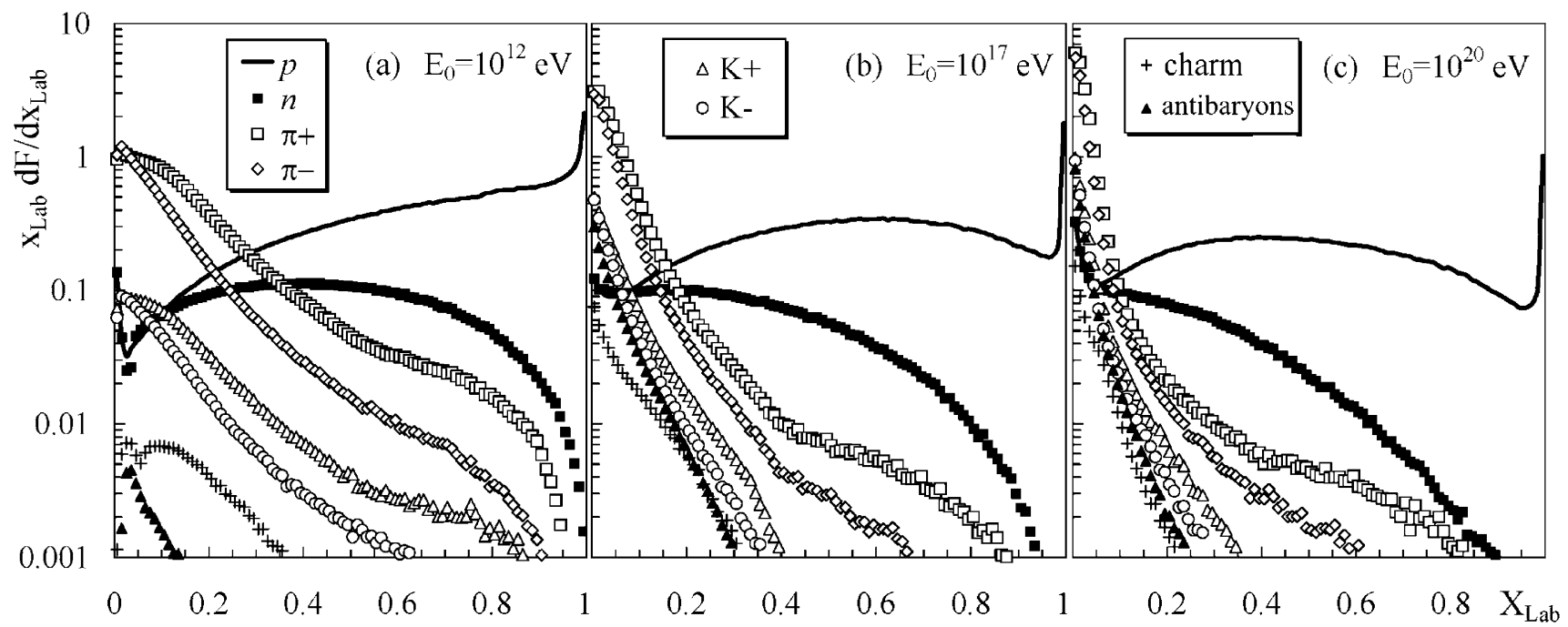

Fig. 2.12 $x_{\mathrm{Lab}} d F / d x_{\mathrm{Lab}}$ distributions simulated by FANSY 1.0 in $p N$ interactions at $E_{0}=10^{12}$ (a), $10^{17}$ (b), and $10^{20}$ (c) eV for protons (curve), neutrons $(\mathbf{\square}), \pi^{+}(\square), \pi^{-}(\diamond), K^{+}(\triangle), K^{-}(O)$, charmed particles $(+)$, antibaryons $(\mathbf{\Delta})$ 


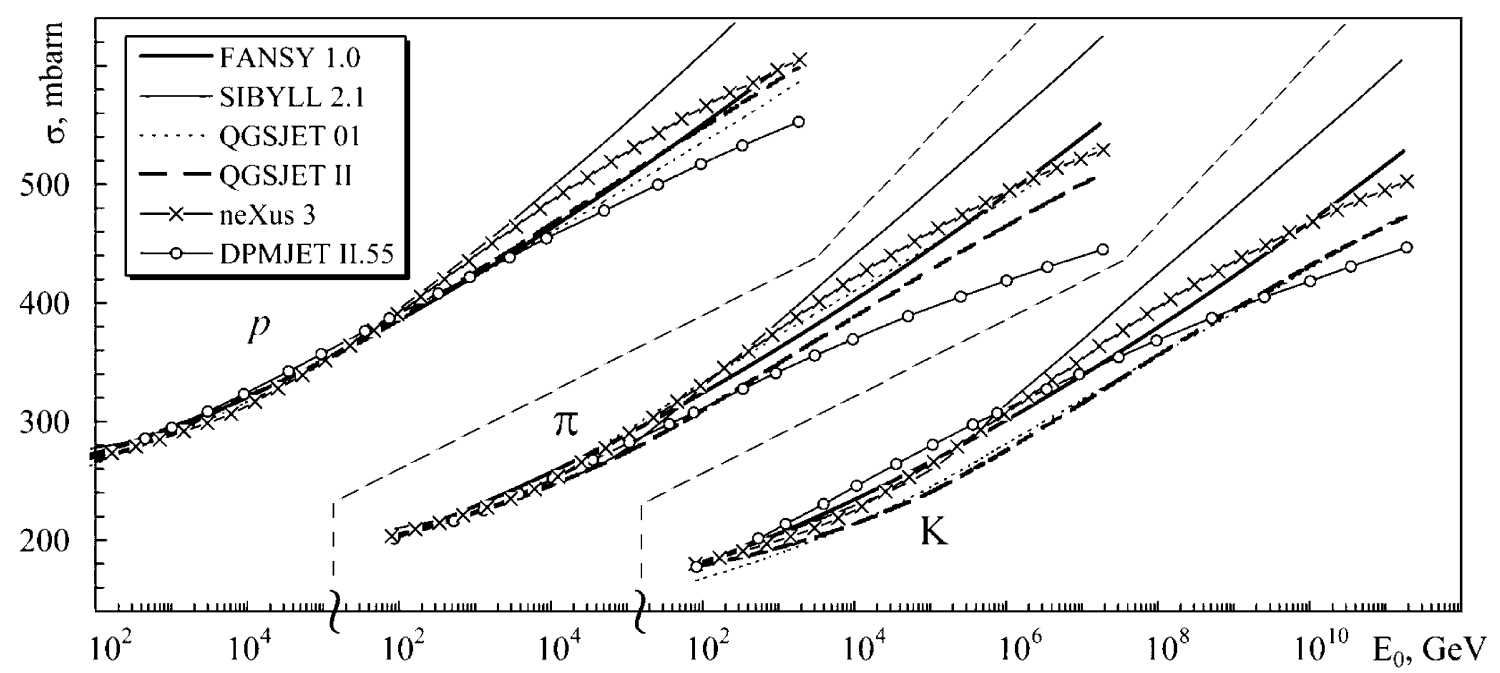

Fig. 2.13 Lab energy dependence of $p$-air, $\pi$-air, and $K$-air cross sections used by FANSY and other models

\section{Simulation of coplanar particle generation}

\subsection{Qualitative description of simulation algorithm}

Unfortunately, all the commonly used well-developed hadronization models deal with a one-dimensional colored field structure, and this is an important part of the modern models. While considering the angular momentum conservation problem with respect to particle generation many years ago, E. Fermi [30] neglected it “... to simplify the mathematics" as well as R. Hagedorn [31], who noted that "this whole question, though of practical importance, seems to be still not understood. ... So at present it seems most reasonable to disregard angular momentum at all...". Unfortunately, this tendency to simplify theoretical calculations concerning this problem dominates until now, although energies under consideration increased since Fermi and Hagedorn's time by many orders of magnitude. Some preliminary ideas related to this aspect of particle generation were only proposed in [15]. As a result, to analyze the observed coplanarity phenomenon, we can construct only quite arbitrary models without any theoretical basis.

While sticking to a semiclassical framework, the following naive picture could be imagined in the c.m.s. of colliding hadrons. The particle generation process is not instant and develops in time and space through appearance of some system (say, a few correlated QGSs) which must have an angular momentum. Let it be initially distributed proportionally to the distance from the string center and the QGS tension be uniformly distributed over the string.

In what follows, the QGS parts adjoining the projectile hadrons (which move rectilinearly in opposite directions) tend to go aside from the hadrons due to the angular motion and angular momentum conservation. Due to these factors, the tension in these ranges becomes higher than the average string tension. So, these parts are the first to rupture and fragment into hadrons.

The subsequent rupture processes run from the string ends to its center. As it takes a certain time, each next disruption takes place after an additional string turn that leads to the coplanar particle generation (CPG) with higher $p_{t}^{\text {copl }}$ and lower $p_{L}$ values. However, starting with a moment, the $p_{t}^{\text {copl }}$ value stops its growth and begins to decrease as the angular momentum begins to decrease due to the approach to the QGS center. Besides, the QGS' angular motion decreases as a whole due to the continued motion of the projectiles away. Naturally, conditions of slowdown and turn-out of the CPG process appear more and more late with increasing energy of collision as well as energy transferred into the rotating system.

This picture is to be symmetrical in c.m.s. in the case of interaction of similar hadrons. In the case of hadronnucleus interaction, the symmetry is broken, both in a kinematic sense and with respect to parameters of the generated temporary rotating system whose features, most likely, differ in ranges adjoining to the projectile hadron and target nucleus. Besides, the energy transferred into secondaries is higher as compared with $p p$ interactions at the same Lab energy value, although the influence of these factors on CPG processes is unknown.

Let us stress that this work does not pretend to a theoretical description of the process under consideration and proposes only a phenomenological tool to study this phenomenon and formulate goals of future theoretical efforts. The above-described oversimplified picture is undoubtedly too naive and, quite possible, is remotely related to the real physical process. However, we would like to stress that the 
below-discussed high- $p_{t}$ processes seem to be quite natural from the semiclassical physics standpoint.

Signatures of the CPG observed in mountain-based experiments correspond to hadron-nucleus interaction energies being as high as $\sqrt{s} \gtrsim 4 \mathrm{TeV}$, i.e., $E_{0} \gtrsim 10^{16} \mathrm{eV}$ [11]. It means that at these relatively low energies the CPG cross section is already comparable with the proton's inelastic one and can present its large fraction. However, we do not know the exact threshold of this effect, peculiarities of its energy dependence, dependence on target nucleus mass, and, as a result, the influence of all these factors on cascade development features. So, different CPG versions are considered below.

The following empirical procedure is applied to provide the necessary $p_{L}-p_{t}^{\text {copl }}$ specific coplanarity-plane correlation mentioned in Introduction. First of all, an interaction is simulated through the traditional QGSJ channel. The CPG is simulated, if the energy transferred by colliding hadrons in their c.m.s. into secondary particles, $E_{\mathrm{sec}}$, is higher than some threshold value, namely, $E_{\mathrm{thr}}^{\mathrm{copl}}=250 \mathrm{GeV}$. If so, particles are primarily ordered in descending energies. Thereupon, values of additional transverse momentum, $\Delta p_{t}^{\text {copl }}$, are sampled for all particles starting from the second one. $\Delta p_{t i}^{\text {copl }}$ values are calculated by an empirical expression, namely, $\Delta p_{t i}^{\text {copl }}=p_{t 0}^{\text {copl }} \cdot\left\{1-\exp \left(\sqrt{x_{i}}\right)\right\}$, where $x_{i}=p_{\mathrm{Li}} / p_{\mathrm{Li}-1} ; p_{t 0}^{\text {copl }}$ depends on model and rises with $E_{\mathrm{sec}}$. In doing so, $p_{t i}^{\mathrm{copl}}=p_{t i-1}^{\mathrm{copl}}+\Delta p_{t i}^{\mathrm{copl}}$.

Starting from some $n$-th particle, the total energy of the remaining secondaries, becomes less than some critical value, $E_{\text {crit }}=0.5 E_{\mathrm{sec}}$. If so, $\Delta p_{t}^{\mathrm{copl}}$ becomes negative and $p_{t}^{\text {copl }}$ begins to decrease and approach traditional values.

Such a rather strange and brute-force empirical algorithm is used to provide the specific coplanarity-plane correlation between longitudinal and transverse momenta of particles (the lower is $p_{L}$, the higher is $p_{t}^{\text {copl }}$ ) and, as a result, fanlike lateral emission of secondaries. The fact is that the use of another way, say, $p_{t} \propto p_{L}$-like linear proportionality, re- alized in the case of generation of narrow high-energy jets, results in formation of beams of particles, not a fan. As a whole, the above-described algorithm does not conflict with the qualitative picture of coplanar particle generation considered in the beginning of this subsection.

\subsection{Coplanarity-related characteristics and parameters}

First of all, let us stress that the CPG-originated increase of $\left\langle p_{t}\left(x_{\mathrm{F}, \mathrm{Lab}}\right)\right\rangle$ takes place only on a coplanarity plane. In the orthogonal plane, $\left\langle p_{t}\right\rangle$ distributions are traditional.

Figure 3.1 displays c.m.s. charged-particle pseudorapidity distributions, $d n_{c h} / d \eta$, realized by FANSY's $Q G S J$, feeble, weak, and strong versions in $p p$ NSD interactions at $E_{0}=10^{15}, 10^{16}, 10^{17}, 10^{19} \mathrm{eV}$. Obviously, distributions are drastically different at $E_{0} \gtrsim 10^{16} \mathrm{eV}$.

Figure 3.2 shows c.m.s. dependence of charged-particle average transverse momentum on $x_{\mathrm{F}}, p_{t}^{c h}\left(x_{\mathrm{F}}\right)$, realized by $Q G S J$, feeble, weak, strong versions at $E_{0}=10^{16}, 10^{17}$, and $10^{19} \mathrm{eV}$ in $p p$ NSD interactions. No difference between the versions takes place at $E_{0} \leq 10^{15} \mathrm{eV}$. At higher energies, these distributions differ very strongly.

Figure 3.3 presents corresponding Lab $p N$-interaction distributions at $E_{0}=10^{16}, 10^{17}$, and $10^{19} \mathrm{eV}$ which differ from those shown in Fig. 3.2 and lie higher as compared with $p p$ interactions. This difference is mainly caused (in the framework of the model) by higher energy values transferred into secondary particles in $p N$ interactions. The dip at $x_{\mathrm{Lab}} \gtrsim 0.95$ is caused by the contribution of diffraction processes taken into account in this case.

Let us note that the range $E_{0}=10^{16}-10^{17}$ is that of effective PCR proton energies responsible for the observable coplanarity phenomenon found in $\gamma$-ray families.

Figure 3.4 displays c.m.s. dependence of average transverse momentum of charged particles on pseudorapidity, $\left\langle p_{t}(\eta)\right\rangle$, realized by $Q G S J$, feeble, weak, strong versions at energies of $10^{15}, 10^{16}, 10^{17}$, and $10^{19} \mathrm{eV}$ in $p p$ NSD interactions. The analogous Lab dependences realized
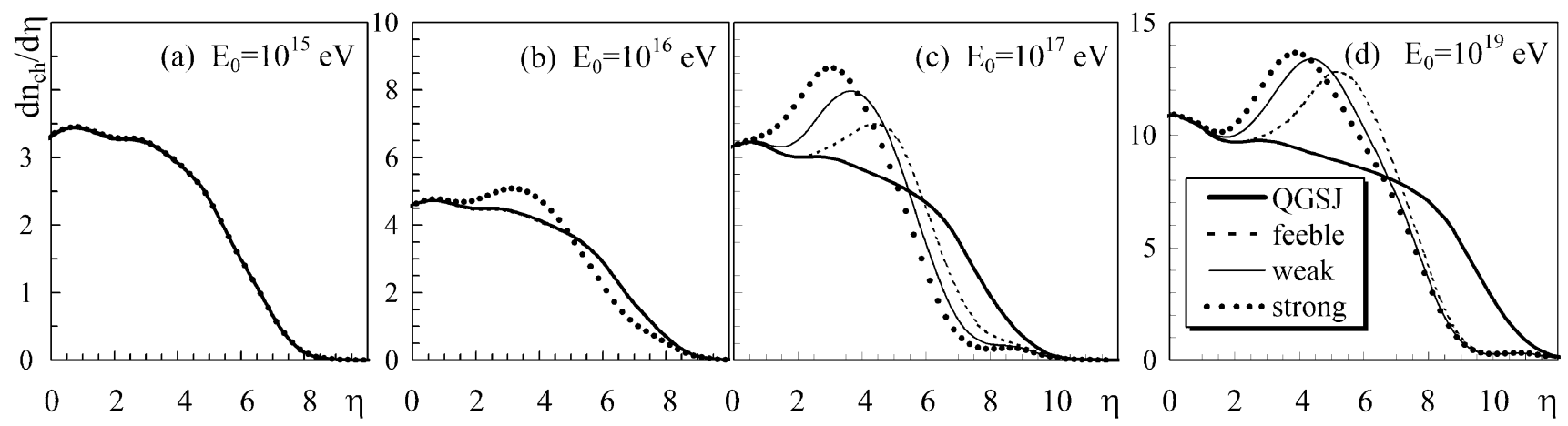

Fig. 3.1 C.m.s. charged-particle pseudorapidity distributions, $d n_{\mathrm{ch}} / d \eta$, in $p p$ NSD interactions at energies of (a) $10^{15} \mathrm{eV},(\mathbf{b}) 10^{16} \mathrm{eV},(\mathbf{c}) 10^{17} \mathrm{eV}$, (d) $10^{19} \mathrm{eV}$ simulated by QGSJ (thick line), feeble (dotted line), weak (thin line), strong (points) 
by FANSY in $p N$ interactions (accounting for the kinematic shifting along the $\eta$ axis) demonstrates more strong energy-dependent variation, i.e., similarly to the difference in $\left\langle p_{t}^{\text {copl }}\left(x_{\mathrm{F}}\right)\right\rangle$ correlation between $p p$ and $p N$ interactions.

Figure 3.5 presents energy dependence of average transverse momentum of positive pions, $\left\langle p_{t}^{\pi+}\left(E_{0}\right)\right\rangle$, realized by different model versions in $p p$ interactions. It should be underlined that average values of transverse momentum, $\left\langle p_{t}\left(E_{0}\right)\right\rangle$, are much lower then its maximum values, $\left\langle p_{t \text { max }}^{\text {copl }}\left(E_{0}, x_{F}\right)\right\rangle$, realized at $x_{\mathrm{F}} \sim 0.03$ (see Fig. 3.2). It is obviously caused by the fact that the main part of particles are generated in the central kinematic region where $\mathrm{CPG}$ processes are negligible.

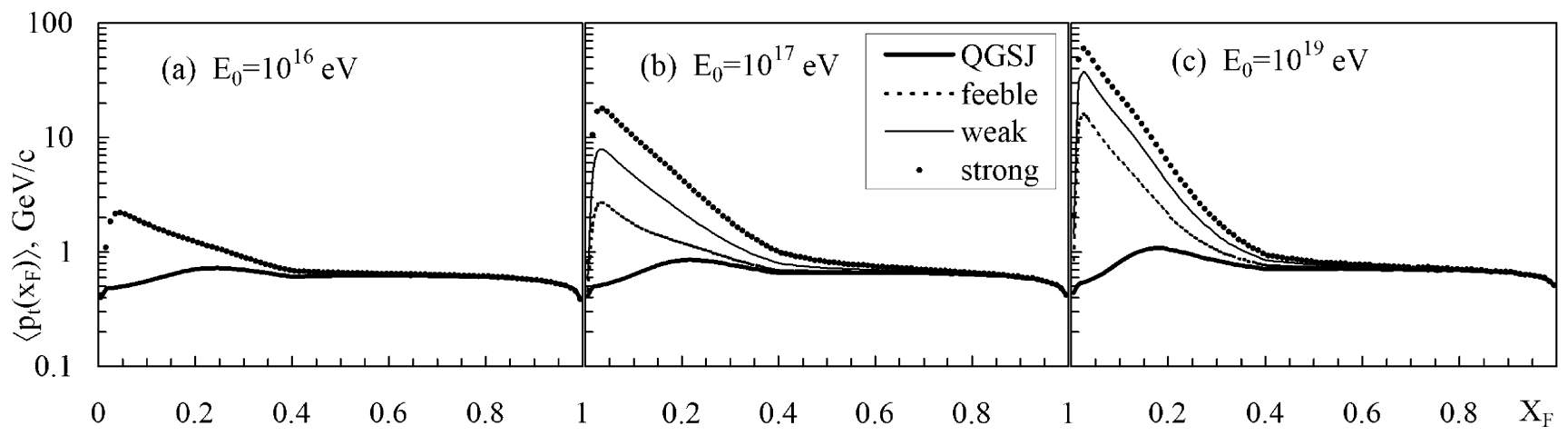

Fig. 3.2 C.m.s. correlations between $x_{\mathrm{F}}$ and $\left\langle p_{t}\left(x_{\mathrm{F}}\right)\right\rangle$, for charged particles realized by FANSY's QGSJ (thick line), feeble (dotted line), weak (thin line), strong (points) versions at (a) $10^{16} \mathrm{eV},(\mathbf{b}) 10^{17} \mathrm{eV}$, (c) $10^{19} \mathrm{eV}$ in $p p$ NSD interactions

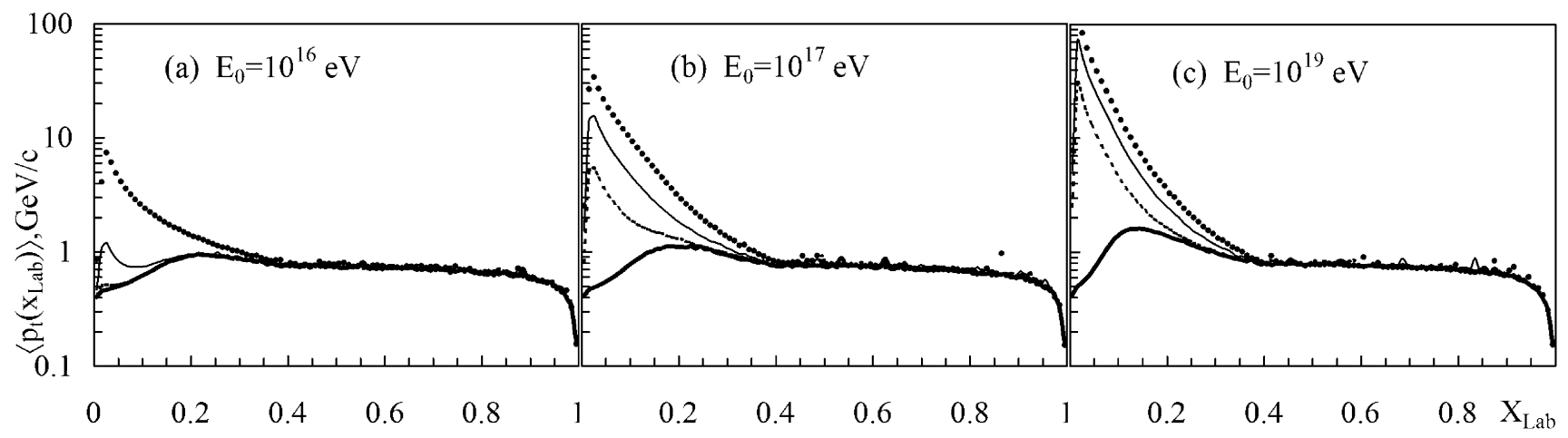

Fig. 3.3 Lab correlation between $x_{\text {Lab }}$ and $\left\langle p_{t}\left(x_{\text {Lab }}\right)\right\rangle$ for charged particles realized by FANSY's versions at (a) $10^{16} \mathrm{eV}$, (b) $10^{17} \mathrm{eV}$, (c) $10^{19} \mathrm{eV}$ in $p N$ interactions. Notations are the same as in Fig. 3.2

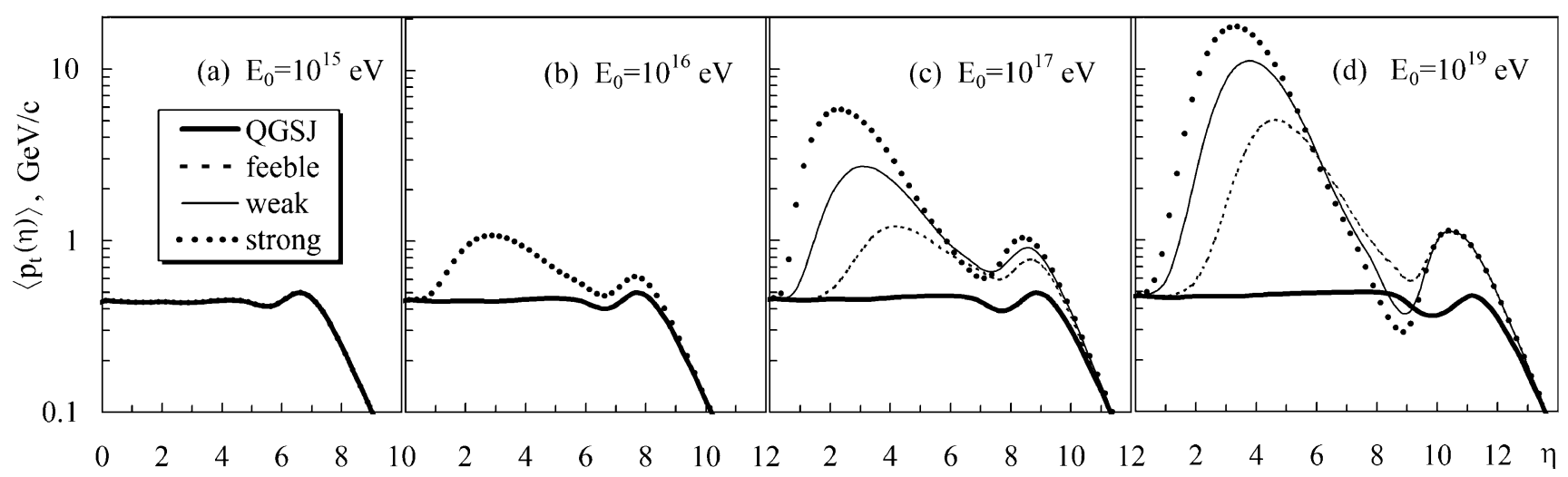

Fig. 3.4 C.m.s. pseudorapidity dependence of $\left\langle p_{t}(\eta)\right\rangle$, for charged particles realized by FANSY's versions at interaction energies of (a) $10^{15}$ eV, (b) $10^{16} \mathrm{eV},(\mathbf{c}) 10^{17} \mathrm{eV},(\mathbf{d}) 10^{19} \mathrm{eV}$ in $p p$ NSD interactions. Notations are the same as in Fig. 3.2 
To analyze the coplanarity of interactions in terms usually applied in [1-4, 6-10], the parameter $\lambda_{N}$ [1] decreasing

from 1 (for $N$ points disposed along a straight line) to $-1 /(N-1)$ (in isotropic cases) is applied. As this parameter has been first defined for $N$ points placed on a plane, coordinates of points of intersection of particles' trajectories with a target plane placed normally to the projectile particle direction are calculated. An event is referred to as coplanar, if the inequality $\lambda_{N} \geq 0.8$ is valid for $N$ most energetic particles generated in this interaction.

Figure 3.6 displays energy dependences of fractions of coplanar events, $F^{\text {copl }}\left(\lambda_{4} \geq 0.8\right)$ (calculated for four most energetic particles) in $p p$ NSD interactions realized by $Q G S J$, feeble, weak, strong versions. Figure 3.7 displays similar Lab dependences realized in $p N$ interactions. The traditional $Q G S J$ version demonstrates an energyindependent behavior with $F\left(\lambda_{4} \geq 0.8\right) \simeq 0.05$, i.e., the fluctuation-caused background value [14] is observed. No doubt, any traditional model will give the same result. Other versions realize different energy-dependent behavior of frac-

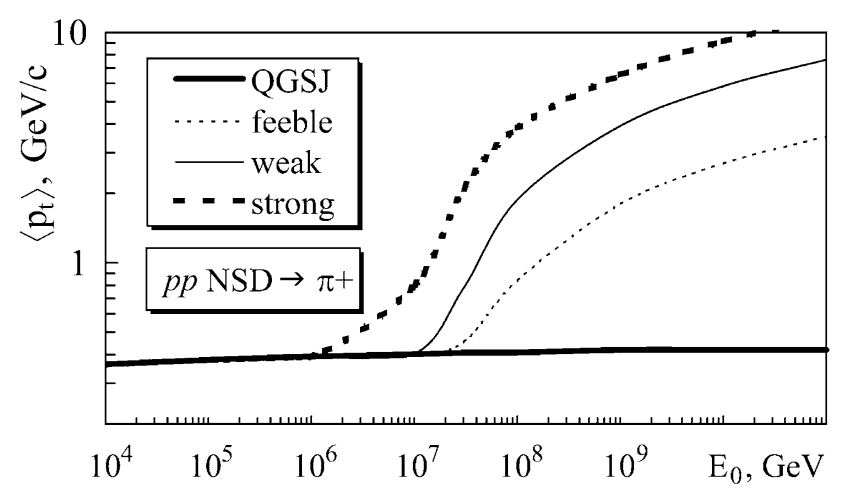

Fig. 3.5 Energy dependence of positive-pion $\left\langle p_{t}^{\pi+}\right\rangle$ realized in $p p$ interactions simulated by QGSJ (thick line), feeble (dotted line), weak (thin line), strong (thick dotted line) versions

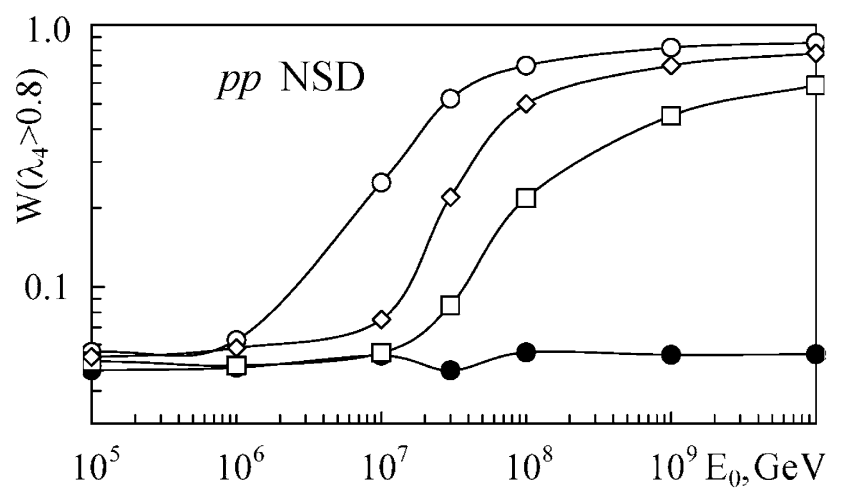

Fig. 3.6 Energy dependence of the fraction of coplanar interactions, $F\left(\lambda_{4} \geq 0.8\right)$, for four most energetic particles in $p p$ interactions realized by FANSY 1.0's $Q G S J(\bullet)$, feeble $(\square)$, weak $(\diamond)$, and strong $(\bigcirc)$ versions

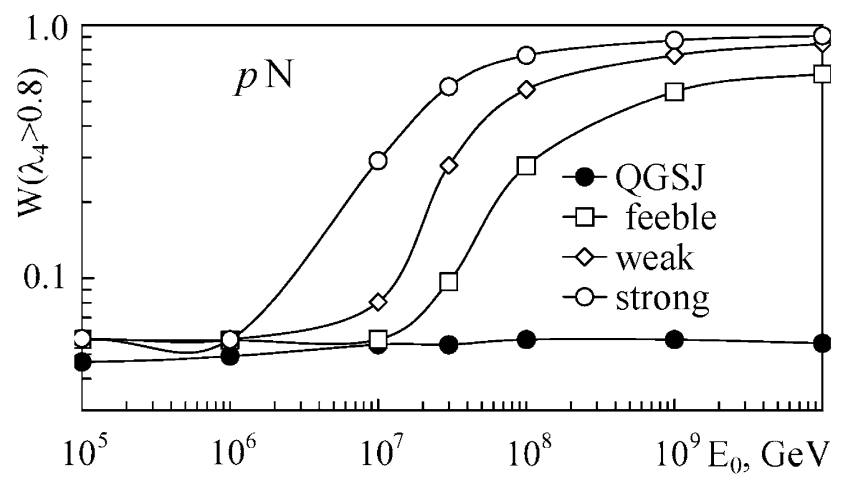

Fig. 3.7 Energy dependence of the fraction of coplanar interactions, $F\left(\lambda_{4} \geq 0.8\right)$, for four most energetic particles in $p N$ interactions realized by FANSY 1.0's $Q G S J(\bullet)$, feeble $(\square)$, weak $(\diamond)$, and strong $(\bigcirc)$ versions

tions of coplanar events. Obviously, the $p N$-interaction coplanarity rises more fast than that in $p p$ interactions.

\section{Comparison with experimental data}

\subsection{Air shower model testing}

As a whole, there is not accelerator experimental data at $\sqrt{s} \gtrsim 1.8 \mathrm{TeV}$ until now. As regards the forward kinematic range $\left(x_{\mathrm{F}} \gtrsim 0.05\right)$, data are already absent at $\sqrt{s}>63 \mathrm{GeV}$. Only cosmic rays can be applied at superhigh energies to test theoretical models.

To test FANSY's longitudinal characteristics, it is natural to compare, first of all, experimental and calculated intensities of different cosmic-ray components produced in the Earth's atmosphere by PCR particles. Figures 4.1 and 4.2 demonstrate spectra of muons and hadrons calculated by using Gaiser and Honda's PCR fit and related to the initial and final stages of air showers, respectively. Spectra predicted by FANSY agree with the experimental hadron spectrum, is not far from experimental muon spectrum and placed in the range of CORSIKA model results.

Another important characteristic related to higher interaction energies $\left(\left\langle E_{0}\right\rangle \gtrsim 10^{15} \mathrm{eV}\right)$ is the intensity of $\gamma$-ray families. Experimental [27] and simulated intensities of $\gamma$ ray families with $\sum E_{\gamma} \geq 100 \mathrm{TeV}$ are $0.54 \pm 0.06$ and $0.62 \pm 0.03\left(\mathrm{~m}^{2} \text { year } \mathrm{sr}\right)^{-1}$, respectively, i.e., a rather good agreement in the error limits is observed.

Thus, FANSY 1.0 gives reasonable results and can be applied for further analysis.

\subsection{Coplanarity in XREC experiments}

First of all, one should emphasize that the real CPG-related coplanarity of $\gamma-h$ families must be lower as compared with single interactions while other things being equal. That 


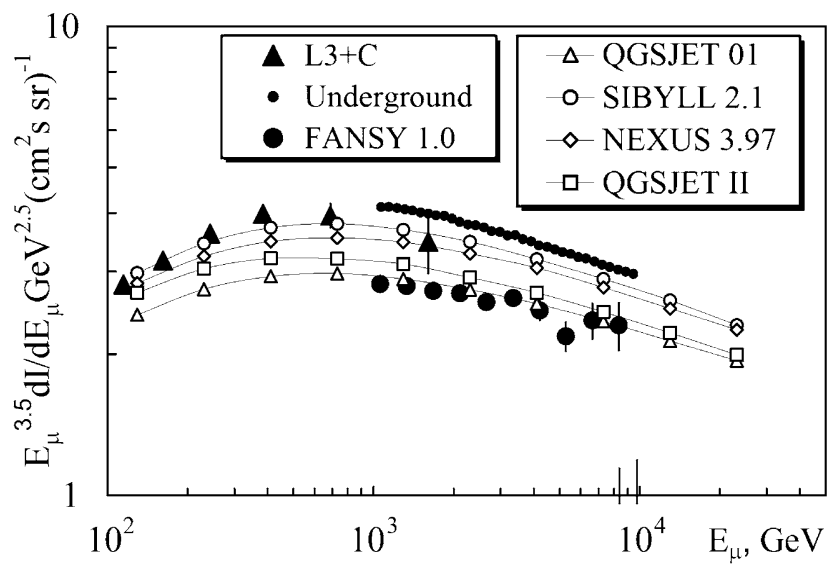

Fig. 4.1 Energy dependence of experimental and model spectra of muons at the sea level

is caused by the destructive role of cascade development following CPG interactions. As a result, at mountain levels it is necessary to separate energy flows in $\gamma$-families, not single particles, with using a so called decascading algorithm $[11,28]$ for the following analysis.

Second, the proton-initiated $\gamma$-ray families dominate among observable events at any, more or less, reliable PCR mass composition due to high-energy threshold of detection and, as a result, lower efficiency of nuclei in formation of families, which decreases quickly with increasing mass of primary nuclei. On the other hand, each nucleus-initiated $\gamma$ ray family is a result of superposition of separated nucleoninitiated cascades. If so, such families are characterized by a lower coplanarity in the framework of any CPG-type model.

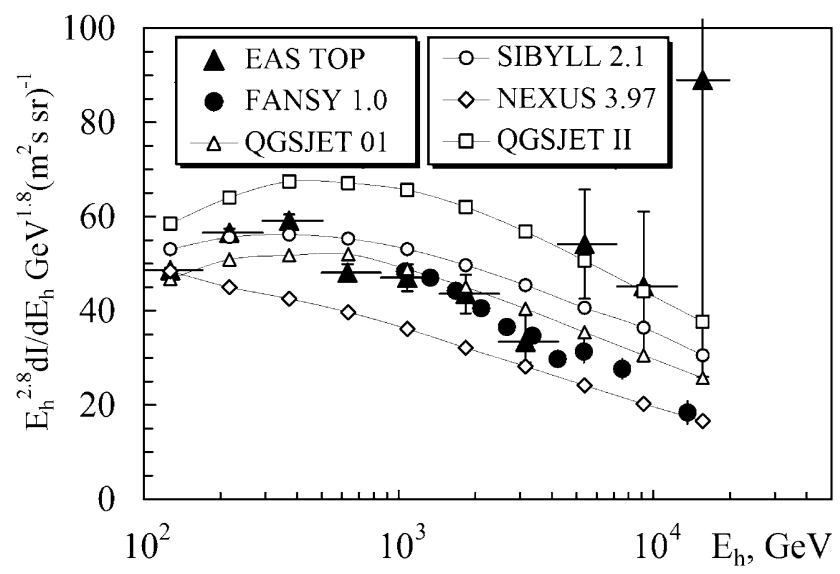

Fig. 4.2 Energy dependence of experimental (EAS-TOP) and model spectra of hadrons at a depth of $840 \mathrm{~g} \cdot \mathrm{cm}^{-2}$

So, we consider below only proton-initiated $\gamma$-families to make the general situation more evident.

Let us remind that a reliable analysis must be only based on all the experimental data accumulated in five independent data sets, namely, Pamir's C-XRECs, Pamir's PbXRECs, Mt.Canbala's Fe-XRECs and two highest-energy stratospheric events, Strana and JF2af2.

Table 4.1 shows selection criteria used to select experimental data, real numbers and relative fractions of coplanar events, $N_{\exp }^{\text {copl }}$ and $F_{\exp }^{\text {copl }}$, observed in each experimental data set. Statistical errors are calculated by the author.

Table 4.2 shows simulated fractions of coplanar events, $F_{\text {sim }}^{\text {copl }}$, found by using different FANSY's versions in accordance with specific selection criteria (for instance, including
Table 4.1 Summary of experimental data. Columns: 1. Data set and type of used XRECs; 2. Criteria for selection of coplanar events; 3 . Number of coplanar families, $N_{\text {exp }}^{\text {copl }}$, and total family number $N_{\text {tot }}$; 4. Fraction of coplanar families, $F_{\exp }^{\text {copl }}$

\begin{tabular}{lllll}
\hline Experimental data set & Selection criteria $\left(\lambda_{N}, \sum E_{\gamma}\right)$ & $N_{\exp }^{\text {copl }}$ from $N_{\text {tot }}$ & $F_{\text {exp }}^{\text {copl }}$ & Ref. \\
\hline Pamir $(\mathrm{Pb}-\mathrm{XREC})$ & $\lambda_{4} \geq 0.8, \sum E_{\gamma} \geq 700 \mathrm{TeV}$ & 6 from 14 & $0.43 \pm 0.13$ & {$[3]$} \\
Pamir $(\mathrm{C}-\mathrm{XREC})$ & $\lambda_{4} \geq 0.8, \sum E_{\gamma} \geq 700 \mathrm{TeV}$ & 5 from 35 & $0.15 \pm 0.05$ & {$[5]$} \\
Mt.Kanbala (Fe-XREC) & $\lambda_{3} \geq 0.8, \sum E_{\gamma} \geq 500 \mathrm{TeV}$ & 6 from 12 & $0.5 \pm 0.13$ & {$[6]$} \\
the Strana & $\lambda_{4}=0.99, \sum E_{\gamma} \simeq 1500 \mathrm{TeV}$ & 1 & 1 & {$[12,13]$} \\
the JF2af2 & $\lambda_{4}=0.998, \sum E_{\gamma} \simeq 1500 \mathrm{TeV}$ & 1 & 1 & {$[9,10]$} \\
\hline
\end{tabular}

Table 4.2 Simulated data on fraction of coplanar $\gamma$-ray families. Columns: 1 . Experimental data sets and types of used XRECs; 2. Selection criteria; 3-6. Fraction of coplanar families, $F_{\text {sim }}^{\text {copl }}$, calculated by $Q G S J$ (3), feeble (4), weak (5), strong (6)

\begin{tabular}{|c|c|c|c|c|c|}
\hline \multirow{2}{*}{$\begin{array}{l}\text { Experimental } \\
\text { data set }\end{array}$} & \multirow[t]{2}{*}{ Selection criteria } & \multicolumn{4}{|l|}{$F_{\text {sim }}^{\mathrm{copl}}$} \\
\hline & & $Q G S J$ & feeble & weak & strong \\
\hline $\operatorname{Pamir}(\mathrm{Pb})$ & $\lambda_{4} \geq 0.8, \sum E_{\gamma} \geq 700 \mathrm{TeV}$ & $0.041 \pm 0.004$ & $0.057 \pm 0.005$ & $0.082 \pm 0.006$ & $0.14 \pm 0.01$ \\
\hline $\operatorname{Pamir}(\mathrm{C})$ & $\lambda_{4} \geq 0.8, \sum E_{\gamma} \geq 700 \mathrm{TeV}$ & $0.049 \pm 0.004$ & $0.058 \pm 0.005$ & $0.083 \pm 0.006$ & $0.15 \pm 0.01$ \\
\hline Mt.Kanbala $(\mathrm{Fe})$ & $\lambda_{3} \geq 0.8, \sum E_{\gamma} \geq 500 \mathrm{TeV}$ & $0.18 \pm 0.01$ & $0.22 \pm 0.01$ & $0.24 \pm 0.01$ & $0.27 \pm 0.01$ \\
\hline the Strana & $\lambda_{4} \geq 0.99, \sum E_{\gamma} \geq 1400 \mathrm{TeV}$ & $0.0026 \pm 0.0003$ & $0.0046 \pm 0.0004$ & $0.017 \pm 0.001$ & $0.093 \pm 0.002$ \\
\hline \multirow[t]{2}{*}{ the $J F 2 a f 2$} & $\lambda_{4} \geq 0.995, \sum E_{\gamma} \geq 1400 \mathrm{TeV}$ & $(9 \pm 3) \times 10^{-4}$ & $0.0027 \pm 0.0005$ & $0.010 \pm 0.001$ & $0.030 \pm 0.002$ \\
\hline & $\lambda_{38} \geq 0.95, \sum E_{\gamma} \geq 1400 \mathrm{TeV}$ & $\ll 10^{-9}$ & $\ll 10^{-6}$ & $\ll 10^{-4}$ & $\lesssim 10^{-4}$ \\
\hline
\end{tabular}


the decascading algorithm with $z_{c}=5 \mathrm{TeV} \cdot \mathrm{cm}$ for Pamir's XRECs [11, 28]).

One can see that the simulated fraction magnitude rises with increasing model's radicalism. However, all the models give lower values as compared with experimental data. Only the strong version give, more or less, appropriate values.

Let us attempt to find estimates of probabilities, $w_{i}$, to gain the real (or larger) experimental number of coplanar families in the framework of different FANSY's versions. Results are shown in Table 4.3.

While assuming the statistical independence of experimental data by different experimental sets and avoiding discussions on correctness of the following operation, let us multiply the values found for data sets and get semiquantitative evaluative values characterizing the probability to observe the total experimental data set in the framework of each FANSY's version, $W_{\mathrm{tot}}=\prod_{i=1}^{5} w_{i}$. In doing so for QGSJ, feeble, weak, and strong versions, we have as low values as $\sim 10^{-14}, \sim 4 \times 10^{-12}, \sim 7 \times 10^{-9}, \sim 12 \times 10^{-6}$, respectively. Statistical errors are of about one order of magnitude.

Comparison of these model results with experimental data given in Tables 4.2 and 4.3 demonstrates the necessity of a coplanar particle generation process. The feeble and weak versions seems to be too weak to explain cosmicray experimental data. The strong model seems to be more appropriate. Although it also gives a rather lower average values of the coplanar-event fraction as compared with the total set of experimental data, the probability is already significant and cannot be neglected.

However, it should be underlined that in the case of CPG process the higher is the number of particles, the clearer is the coplanarity phenomenon. So, the experimental extreme coplanarity of $38 \gamma$-rays in the stratospheric JF2af2 event (being much less corrupted by cascade development) requires a special discussion. The corresponding value $\lambda_{38}$ is not published and can be only estimated. While accounting for a high correlation coefficient value, $\beta_{38}=0.9993$, and a strong correlation between $\beta_{N}$ and $\lambda_{N}$ values at $\lambda_{N} \sim \beta_{N} \sim 1, N \gg 1$, we can assume with high confidence that, say, $\lambda_{38} \geq 0.95$. In this case, any traditional model gives $w_{i}\left(\lambda_{38} \geq 0.95\right) \ll 10^{-9}$. If so, the total probability value found in the framework of FANSY/QGSJ is as low as $W_{\mathrm{tot}}^{*}=\prod_{i=1}^{5}{ }^{*} w_{i} \ll 10^{-20}$ ! Thus, the explanation of the alignment phenomenon with fluctuations of cascade development in the framework of such a model seems to be actually improbable. The feeble, weak, and strong versions give $W_{\text {tot }}^{*} \ll 10^{-18}, \ll 10^{-13}$, and $\lesssim 10^{-9}$, respectively.

A similar situation takes place in the case of the Strana event. The experimental anisotropy of 33 particles in its central region estimated by using different parameters is much higher than that simulated, for instance, by QGSJET model [29].

\subsection{Coplanarity and EAS experiments}

As regards relations between EAS ground-based experiments and the above-mentioned experimental and simulated results, the most important consequence is a possible influence of the coplanarity phenomenon on EAS lateral features used to study the PCR composition at super-high energies. As is shown in [14] on the basis of a rather primitive and moderate CPG model, some characteristics of protoninitiated EAS's can imitate those of light nucleus-initiated ones. Until now, contradictory EAS experimental data do not permit to describe the PCR composition by some selfconsisting way. It cannot be excluded that an accomplished model capable to describe all the set of experimental data cannot be constructed without including the coplanarity phenomenon.

The only work [26] devoted to search for experimental signs of coplanarity phenomenon in EAS hadrons by a ground-based array has not found indications in favor of this effect. However, two censorious remarks are to be made. First, a too simplified simulation procedure was really applied to analyze the coplanarity problem, namely, transverse momenta of all particles at all energies were enlarged by two times and no coplanar effects and/or their energy dependence were assumed. As a result, the obtained result is not unexpected, namely, simulated EAS lateral distribution of hadrons with rather low energies, $E_{h}>100 \mathrm{GeV}$, become wider. However, if the CPG process shows up at very high interaction energies, $E_{0} \sim 10^{16} \mathrm{eV}$, then it is necessary to analyze the energy flows with energies higher of several tens
Table 4.3 Probabilities to observe real (or larger) experimental number of coplanar families in accordance with FANSY's versions.

Columns: 1. Experimental data set; $2-5$. Probability $w_{i}$ calculated with $Q G S J$ (2), feeble (3), weak (4), strong (5)

\begin{tabular}{|c|c|c|c|c|}
\hline \multirow[t]{2}{*}{ Experimental data } & \multicolumn{4}{|l|}{$w_{i}$} \\
\hline & $Q G S J$ & feeble & weak & strong \\
\hline $\operatorname{Pamir}(\mathrm{Pb})$ & $(11 \pm 7) \times 10^{-6}$ & $(7 \pm 4) \times 10^{-5}$ & $(5 \pm 2) \times 10^{-4}$ & $0.008 \pm 0.003$ \\
\hline Pamir (C) & $0.026 \pm 0.010$ & $0.050 \pm 0.021$ & $0.16 \pm 0.04$ & $0.62 \pm 0.06$ \\
\hline Mt.Kanbala & $0.012 \pm 0.004$ & $0.030 \pm 0.007$ & $0.045 \pm 0.010$ & $0.076 \pm 0.013$ \\
\hline the Strana & $0.0026 \pm 0.0003$ & $0.0046 \pm 0.0004$ & $0.017 \pm 0.001$ & $0.093 \pm 0.002$ \\
\hline the JF2af2 $\left(\lambda_{4} \geq 0.995\right)$ & $(9 \pm 3) \times 10^{-4}$ & $0.0027 \pm 0.0005$ & $0.010 \pm 0.001$ & $0.030 \pm 0.002$ \\
\hline$\left(\lambda_{38} \geq 0.95\right)$ & $\ll 10^{-9}$ & $\ll 10^{-6}$ & $\ll 10^{-4}$ & $\lesssim 10^{-4}$ \\
\hline
\end{tabular}


of $\mathrm{TeV}$ in the central EAS core range with a specific dimension of a few centimeters [14], not single low-energy hadrons at large distances from the core. Obviously, today EAS arrays are unsuitable for such fine studies. The second important remark is that there is no sense to search for coplanarity effects at the sea level in any case since even a high coplanarity degree of secondary particles taking place immediately after a superhigh-energy interaction is quickly destroyed by cascade-development processes. This effect is to be only studied in high-altitude mountain or stratospheric experiments.

Let us emphasize that the dependence of EAS lateral features on the coplanarity process is not too evident due to the specific kinematics of this phenomenon. The fast growth of transverse momentum begins at very high energies and is related to most energetic particles which move inside a narrow solid angle. Besides, the transverse momentum rises more slowly than energy increases. As a result, the specific solid angle related to the most energetic particles with large transverse momenta decreases with increasing energy in any case. The observed EAS lateral distribution is mainly determined by lower-energy interactions described by traditional models. Thus, accurate simulations are required to understand the real influence of the coplanar particle generation on EAS lateral characteristics, which are much less sensitive to variations of interaction characteristics as comparable with $\gamma-h$ families.

\subsection{Coplanarity and collider experiments}

It would be most attractive to test the above-described model in LHC experiments. As Fig. 3.6 shows, the LHC energy range is appropriate to study the CPG process. However, it is necessary to solve a number of difficulties caused by collider's specific kinematics of experiments and high beam luminosity. First of all, this effect can be study only by eventby-event way, i.e., at very low collider luminosity. Second, the effect is related, on the one hand, to a range of large $x_{\mathrm{F}}$ values and, on the second hand, to high transverse momenta.

Figure 4.3 shows pseudorapidity dependence of transverse momentum $\left\langle p_{t}(\eta)\right\rangle$ at $\sqrt{s}=14 \mathrm{TeV}$ for different FANSY's versions and kinematic ranges exploited by CMS, CASTOR, TOTEM, ZDC, and LHCf experiments.

Obviously, the LHCf and ZDC experiments with characteristic sizes of $h \sim 10 \mathrm{~cm}$, which are aimed at the study of high $x_{\mathrm{F}}$ values and placed at $L=140 \mathrm{~m}$ from interaction points, are capable of detecting only particles whose transverse and longitudinal momenta satisfy the inequality $p_{t} / p_{z} \lesssim h / L$, i.e., particles with $p_{t} \lesssim(h / L) \cdot(\sqrt{s} / 2) \cdot x_{\mathrm{F}} \simeq$ $x_{\mathrm{F}} \cdot 5 \mathrm{GeV} / c$. It means that in the range of a real coplanarity effect, say, at $x_{\mathrm{F}} \sim 0.2$, only particles with $p_{t}<1.0 \mathrm{GeV} / c$ can be observed. This is illustrated by Fig. 4.4 which shows two examples of target diagrams placed at a distance of
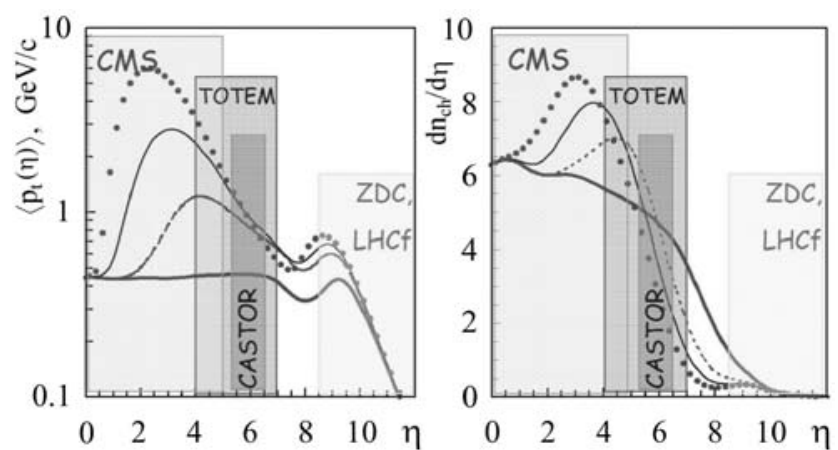

Fig. 4.3 Pseudorapidity dependence of charged-particle $p_{t}$ (left) and $d n_{\mathrm{ch}} / d \eta$ distribution (right) at $\sqrt{s}=14 \mathrm{TeV}$ in $p p$ NSD interactions. Shaded areas show kinematic ranges studied by CMS, CASTOR, TOTEM, ZDC, and LHCf experiments. Notations are the same as in Fig. 3.4

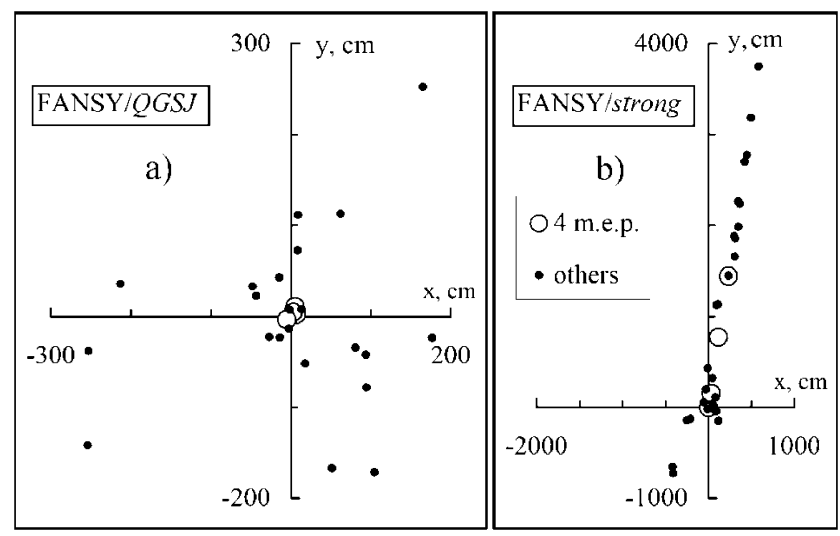

Fig. 4.4 Target diagram at $140 \mathrm{~m}$ from the interaction point for one $p p$ interaction realized by (a) $Q G S J$ and (b) strong versions at $\sqrt{s}=14 \mathrm{TeV}$. Circles denote four most energetic particles, points show other lower-energy particles

$140 \mathrm{~m}$ from the interaction point for a single interaction realized by FANSY's (a) $Q G S J$ and (b) strong versions at LHC energy. Multiplicity, longitudinal momenta are the same in both the cases which differ only in transverse momenta. Obviously, detection of four most energetic particles shown by circle requires a much larger detector dimensions.

The CMS, CASTOR and TOTEM experiments could be more promising in this sense as their operation pseudorapidity ranges are $-5<\eta<5,5.25<\eta<6.5$, and $4<\eta<7$, respectively, while some CPG effects could manifest themselves in these ranges (at least, in the framework of the model under consideration). In any case these experiments can give information on $\left\langle p_{t}\right\rangle$, while feeble, weak, and strong versions predict different $\left\langle p_{t}^{\pi+}\right\rangle$ values at LHC energies, in particular, $\left\langle p_{t}\right\rangle=2.4,4.5$, and $5.6 \mathrm{GeV} / c$, respectively (see Fig. 3.5). 


\subsection{Theoretical approach to coplanarity}

It should be stressed that the most important problem is to develop the theory of coplanar particle generation including, for instance, the mechanism of transformation of the QGS angular momentum into transverse momenta of particles or some another process. In doing so, it is important to understand the difference between hadron-nucleus and hadron-baryon interactions. It cannot be excluded that this phenomenon first manifests itself just in hadron-nucleus interactions due to some nucleus effects.

Several approaches have been proposed [15-19] until now. One cannot exclude other approaches. However, any successful theory are to take into account last three CPG features listed in Introduction, namely, high cross section, specific correlation between $p_{L}$ and $p_{t}^{\text {copl }}$, and a causal relationship of CPG with most energetic secondary particles.

\section{Conclusion}

A computer FANSY 1.0 code is developed to study the coplanar particle generation (CPG) phenomenon.

FANSY 1.0 includes a few versions, namely, $Q G S J$ based on QGS/QCD/minijets concepts as well as feeble, weak and strong versions which apply different energy dependences of CPG parameters and predict significant energy dependence of average transverse momentum, $\left\langle p_{t}\right\rangle$, and maximum $\left\langle p_{t}^{\text {copl }}\left(\eta, x_{\mathrm{F}}\right)\right\rangle$ magnitudes as compared with QGSM-based models.

Comparison of these versions' results with experimental data demonstrate the necessity of introducing a CPG process.

The feeble and weak versions seem to be too weak to explain cosmic-ray experimental data. The strong version seems to be most appropriate.

It would be very desirable to test the above-described model in LHC experiments although it is rather difficult due to collider's specific kinematics and high luminosity. In any case a large average transverse momentum (3-4 GeV/c) would be measured at $\sqrt{s}=14 \mathrm{TeV}$.

The most important goal is to develop the theory of coplanar particle generation.

Acknowledgements This work is partially supported by the RFBR, projects 06-02-16606, 06-02-16969; and Ministry of Education and Science, project SS 959.2008.2.

\section{References}

1. A. Borisov et al. (Pamir Collaboration), in Proc. of 4th Int. Symp. on Very High Energy Cosmic Ray Interactions, Beijing, ed. by D. Linkai et al. (1986), pp. 4-29

2. I.P. Ivanenko et al., JETP Lett. 50, 2125 (1992)

3. V.V. Kopenkin et al., Phys. Rev. D 52, 2766 (1995)

4. Pamir Collaboration, Preprint INP MSU (1989) no. 89-67/144

5. A.S. Borisov et al., Nucl. Phys. B (Proc. Suppl.) 97, 118 (2001)

6. L. Xue et al., in Proc. 26th Int. Cosmic Ray Conf., Salt Lake City, vol. 1 (1999), p. 127

7. A.V. Apanasenko et al., in Proc. 15th Int. Cosmic Ray Conf., Plovdiv, vol. 7 (1977), p. 220

8. A.K. Managadze et al., in Proc. 27th Int. Cosmic Ray Conf., Hamburg, vol. 1 (2001), p. 1426

9. J.N. Capdevielle, J. Phys. G 14, 503 (1988)

10. J.N. Capdevielle et al., in Proc. 30th Int. Cosmic Ray Conf., Merida, HE, vol. 120 (2007)

11. A.S. Borisov et al., Nucl. Phys. B (Proc. Suppl. A) 75, 144 (1999)

12. A.K. Managadze et al., in Proc. 29th Int. Cosmic Ray Conf., Pune, vol. 9 (2005), p. 81

13. A.K. Managadze et al., Phys. At. Nucl. 70(1), 184 (2007)

14. R.A. Mukhamedshin, J. High Energy Phys. 5, 049 (2005)

15. T. Wibig, hep-ph/0003230

16. I.I. Royzen, Mod. Phys. Lett. A 9(38), 3517 (1994)

17. J.N. Capdevielle et al., in Proc. 28th Int. Cosmic Ray Conf., Tsukuba, vol. 1 (2003), p. 1599

18. T.S. Yuldashbaev, Kh. Nuritdinov, V.M. Chudakov, Nuovo Cimento C 24, 569 (2001)

19. R.A. Mukhamedshin, Nucl. Phys. B (Proc. Suppl. A) 75, 141 (1999)

20. J. Knapp, D. Heck, G. Schatz, Preprint. Institute of Kernphysic, FZKA 5828, 1996

21. VIHKOS CORSIKA School 2005. http://www-ik.fzk.de/corsika/ corsika-school/

22. G.F. Fedorova, R.A. Mukhamedshin, Bull. Soc. Sci. Lett. Lodz, Ser. Rech. Def. XVI, 137 (1994)

23. T.S. Yuldashbaev et al., in Proc. 26th Int. Cosmic Ray Conf., Salt Lake City, vol. 1 (1999), p. 76

24. Pamir Collaboration, in Proc. 22nd Int. Cosmic Ray Conf., Dublin, vol. 4 (1991), p. 113

25. M. Kalmakhelidze et al., in Proc. 26th Int. Cosmic Ray Conf., Salt Lake City, vol. 1 (1999), p. 100

26. T. Antoni et al., Phys. Rev. D 71, 072002 (2005)

27. A.S. Borisov et al., Nucl. Phys. B (Proc. Suppl.) 175-176, 143 (2008)

28. Pamir Collaboration, Trudy FIAN 154, 3 (1985) (in Russian)

29. V.I. Osedlo et al., in Proc. 30th Int. Cosmic Ray Conf., Merida, HE, no. 617 (2007)

30. E. Fermi, Prog. Theor. Phys. 5, 570 (1950)

31. R. Hagedorn, Nuovo Cimento 15, 434 (1960) 